\title{
Analytic solutions for the one-dimensional compressible Euler equation with heat conduction and with different kind of equations of state
}

Imre Ferenc Barna and László Mátyás 


\title{
ANALYTIC SOLUTIONS FOR THE ONE-DIMENSIONAL COMPRESSIBLE EULER EQUATION WITH HEAT CONDUCTION AND WITH DIFFERENT KIND OF EQUATIONS OF STATE
}

\author{
IMRE FERENC BARNA AND LÁSZLÓ MÁTYÁS
}

Received September 26, 2013

\begin{abstract}
We present analytic self-similar or traveling wave solutions for a one-dimensional coupled system of continuity, compressible Euler and heat conduction equations. Different kind of equations of state are investigated. In certain forms of the equation of state one can arrive to a picture regarding the long time behavior of density and pressure. The impact of these quantities on the evolution of temperature is also discussed.
\end{abstract}

2010 Mathematics Subject Classification: 35L65

Keywords: Euler equation, self-similar Ansatz, traveling waves

\section{INTRODUCTION}

Studying hydrodynamical systems with heat conduction is an important task, both from the application and from the theoretical side as well. There are numerous monographs and textbooks available which investigate three dimensional real fluid mechanics with heat transfer from the engineering point of view [24]. Usually, all the technical questions like the applied numerics, grids are analyzed in depth in these works.

In the following we will investigate the most simple one dimensional system of continuity, compressible and non-viscous Euler and heat conduction equations. We try to find analytic results. Firstly self-similar physically important diffusive solutions are investigated. If such solutions are not found, secondly traveling wave solutions are obtained and discussed.

There are basically two distinct very powerful set of trial functions available to investigate the global properties of the solutions of various single non-linear partial differential equations $[4,11,23,27]$. One is the so-called self-similar solution which describes the intermediate asymptotics of a problem: they hold when the precise initial conditions are no longer important, but before the system has reached its final steady state. They are much simpler than the full solutions, so they are easier to understand and to study in different regions of parameter space. A final reason for 
studying them is that they are solutions of a system of ordinary differential equations and hence do not suffer the extra inherent numerical problems of the full partial differential equations. In some cases self-similar solutions helps to understand diffusion-like properties or the existence of compact supports of the solution.

The other method is the traveling wave Ansatz which gives us a deeper insight into the wave properties of the system like the propagation speed which can be even time dependent.

In our former work the paradox of heat conduction was investigated with the selfsimilar Ansatz [6] and gave a new equation which has a finite propagation speed. Recently, a study about the generalized Cattaneo law is under publication where both the heat conduction coefficient and relaxation time have power law temperature dependence [7]. The results can be continuous or shock wave-like with compact support. With a straightforward generalization of the self-similar Ansatz even a partial differential equation(PDE) system of the 3 dimensional Navier-Stokes equations was successfully investigated [5]. Gilding and Kersner [11] studied large number of nonlinear diffusion-convection problems with the help of traveling waves.

As a beginning, without completeness we mention some analytic studies about hydrodynamical systems which are available in the literature.

Beyond traveling waves and self-similar Ansätze there are various examination techniques available in the literature. Manwai [25] studied the $\mathrm{N}$-dimensional $(N \geq$ 1) radial Navier-Stokes(NS) and Euler equation with different kind of viscosity and pressure dependences and presented analytical blow up solutions. His works are still 1+1 dimensional (one spatial and one time dimension) investigations. Another well established and popular investigation method is based on Lie algebras for which numerous studies are available. Some of them are treating even the three dimensional case, for more see [14]. Unfortunately, no explicit solutions are shown and analyzed there. Fushchich et al. [10] construct a complete set of $\tilde{G}(1,3)$-inequivalent Ansätze of codimension 1 for the NS system and they present 19 different analytical solutions for one or two space dimensions. The last solution is very close to our former result in [5], but they are not identical. Further two and three dimensional studies of the Navier-Stokes equation based on group analytical method were presented by Grassi [13]. They also presented solutions which look almost the same as ours [5], but they consider only two space dimensions.

Recently, Hu et al. [16] presents a study where symmetry reductions and exact solutions of the (2+1)-dimensional NS are investigated. Aristov and Polyanin [2] use various methods like Crocco transformation, generalized separation of variables or the method of functional separation of variables for the NS and present large number of new classes of exact solutions. Sedov in his classical work [23] (Page 120) presented analytic solutions for the three dimensional spherical NS equation where all three velocity components and the pressure have polar angle dependence $(\theta)$ only. 
Even this kind of restricted symmetry led to a non-linear coupled ordinary differential equation system which has a very rich mathematical structure. Some similarity reduction solutions of the two dimensional incompressible NS equation was presented by Xia-Ju [17]. Additional solutions are available for the $2+1$ dimensional NS also via symmetry reduction techniques by [9]. A full three dimensional Lie group analysis is available for the three dimensional Euler equation of gas dynamics, with polytropic EOS, unfortunately without any heat conduction mechanism [20]. Of course one may find numerical methods for solving equations of fluid dynamics [28]. The topic is quite important, there are interesting applications of the equations of fluids in granular matter [12].

To our knowledge there are very few analytic (self-similar or traveling wave) solutions known for any non-linear partial differential equation systems (PDEs) till today. Our experience shows that no such comparative studies are available for fluid mechanics with heat conduction in the literature, which we present on the next pages.

In the next section we outline our starting model and in the later subsections we give various solutions for three different polytropic equation of states (EOS), plus for the virial EOS. If our results meet some other theories (like porous media equation) then the corresponding literature is mentioned. The paper ends with a short summary.

\section{THE BASIC MODEL}

We start with the following one-dimensional compressible and non-viscous fluid with ordinary heat conduction which means the following coupled PDE system of continuity, Euler and heat conduction

$$
\begin{array}{r}
\rho(x, t)_{t}+[\rho(x, t) v(x, t)]_{x}=0, \\
v(x, t)_{t}+v(x, t) v(x, t)_{x}=-\frac{1}{\rho(x, t)} p(x, t)_{x}, \\
T(x, t)_{t}+v(x, t) T(x, t)_{x}=\lambda T(x, t)_{x x},
\end{array}
$$

where $\rho, v, T, P$ are the density, velocity, temperature and pressure field or distribution, respectively. Subscript means partial derivation with respect of time and the $x$ to the Cartesian coordinate. We skip viscous velocity terms in the Euler and in the energy equation as well. As a starting point we include the polytropic EOS $p(x, t)=a \rho(x, t)^{n}$ were $n$ is a real number and $a$ is a material constant and $\lambda$ is the heat conduction coefficient. After the investigation of this basic equation system we will introduce modifications for the equation of state.

In the following we are looking for the solution in the form of the self-similar Ansatz which is well-known from [4,23,27]

$$
V(x, t)=t^{-\alpha} f\left(\frac{x}{t^{\beta}}\right):=t^{-\alpha} f(\eta)
$$


where $V(x, t)$ can be an arbitrary variable of a PDE and $t$ means time and $x$ means spatial dependence. The similarity exponents $\alpha$ and $\beta$ are of primary physical importance since $\alpha$ represents the rate of decay of the magnitude $V(x, t)$, while $\beta$ is the rate of spread (or contraction if $\beta<0$ ) of the space distribution as time goes on. The validity of this self-similar Ansatz also means that the solution has no characteristic time scale. The most powerful result of this Ansatz is the fundamental or Gaussian solution of the Fourier heat conduction equation (or for Fick's diffusion equation) with $\alpha=\beta=1 / 2$. The function $f(\eta)$ is called the shape function.

For our system we use the following notations and shape functions

$$
T(x, t)=t^{-\alpha} f\left(\frac{x}{t^{\beta}}\right), v(x, t)=t^{-\delta} g\left(\frac{x}{t^{\beta}}\right), \rho(x, t)=t^{-\gamma} h\left(\frac{x}{t^{\beta}}\right)
$$

where the new variable is $\eta=x / t^{\beta}$. Calculating all the first time and spatial derivatives of (2.3) and putting them back to (2.1) after some algebra we get the following non-linear ordinary differential equation (ODE) system

$$
\begin{array}{r}
-\gamma h-\frac{1}{2} \eta h^{\prime}+g h^{\prime}+h g^{\prime}=0, \\
-\frac{1}{2} g-\frac{1}{2} \eta g^{\prime}+g g^{\prime}=-a\left(\frac{1+\gamma}{\gamma}\right) h^{\left(\frac{1+\gamma}{\gamma}-2\right)} h^{\prime}, \\
-\alpha f-\frac{1}{2} \eta f^{\prime}+g f^{\prime}=\lambda f^{\prime \prime},
\end{array}
$$

where prime means derivation with respect to $\eta$. Among the initially free parameters $\alpha, \beta, \gamma, n$ we got the following constraints: $\alpha, \gamma$ are still arbitrary but $\beta=\delta=1 / 2$ and $n=(1+\gamma) / \gamma$. The material constants $c, \lambda$ are still independent. Note, that the first and second equations of (2.1) are independent from the third one.

\subsection{Aspects coming from the conservation law of density}

The first equation is a conservation equation (without source term) and if $\gamma=1 / 2$ it can be integrated

$$
\left(\frac{\eta h}{2}\right)^{\prime}=(g h)^{\prime}
$$

giving $g=\eta / 2$ and $g^{\prime}=1 / 2$ when $h \neq 0$ and the integration constant was chosen zero. Plugging this result back into the second equation of (2.1).

$$
-\frac{\eta}{4}=-3 a h h^{\prime}
$$

where $n=(1+\gamma) / \gamma=3$. Which means that only the $p=a \rho^{3}$ ploytropic EOS can give self-similar solutions in this flow system. The solution of (2.6) can be obtained 
via a simple integration giving the following expression

$$
h=\sqrt{\left(\frac{4 \eta^{2}+2 c_{1}}{3 a}\right)}
$$

where $c_{1}$ is the integration constant (we will set it to 0 ) and $a$ is still the material constant of the polytropic EOS. At last, let's investigate the third heat conduction equation. With the knowledge of the velocity its solution becomes quite simple

$$
-\alpha f=\lambda f^{\prime \prime}
$$

The solution is well known

$$
f=c_{2} \cos \left(\frac{\alpha \eta}{\lambda}\right)+c_{3} \sin \left(\frac{\alpha \eta}{\lambda}\right) .
$$

At last, we summarize all our results

$$
g=\frac{\eta}{2}, h=\sqrt{\left(\frac{4 \eta^{2}+2 c_{1}}{3 a}\right)}, f=c_{2} \cos \left(\frac{\alpha \eta}{\lambda}\right)+c_{3} \sin \left(\frac{\alpha \eta}{\lambda}\right)
$$

where $n=3, \gamma=\beta=\delta=1 / 2$ and $\alpha$ is arbitrary. Now the velocity, density and the temperature field read

$$
\begin{aligned}
& v(x, t)=t^{-\delta} g(\eta)=\frac{x}{2 t}, \\
& \rho(x, t)=t^{-\gamma} h(\eta)=t^{-\frac{1}{2}} \sqrt{\frac{4\left(\frac{x}{\sqrt{t}}\right)^{2}+2 c_{1}}{3 c}} \\
& T(x, t)=t^{-\alpha} g(\eta)=t^{-\alpha}\left[c_{2} \cos \left(\frac{\alpha x}{\lambda \sqrt{t}}\right)+c_{3} \sin \left(\frac{\alpha x}{\lambda \sqrt{t}}\right)\right]
\end{aligned}
$$

and the applied polytropic EOS

$$
p=a \rho^{n}=a\left(t^{-\gamma} h(\eta)\right)^{3}=a\left[t^{-\frac{1}{2}} \sqrt{\frac{4\left(\frac{x}{\sqrt{t}}\right)^{2}+2 c_{1}}{3 c}}\right]^{3} .
$$

All the solutions have physical meaning for bounded regions and for sufficiently large times. We will see later, that according to the continuity equation, the velocity field is always a simple function of time and space coordinates, however the temperature distribution have a more complex form. In this case it is a quickly oscillating and decaying function or a slowly oscillating and slowly decaying function. 


\subsection{Aspects of the solution regarding velocity}

We have the possibility to see that in certain cases, when the first equation is integrable, solutions of the system can be found. This was accessible when the pressure is proportional to the third power of the density. Now we try to find solutions when the pressure is proportional to the second power of the density

$$
p=\frac{b}{2} \rho^{2} .
$$

The role of $b$ is to fix the proper physical dimension. In this case the second equation of (2.1) can be written

$$
v_{t}+v v_{x}=-b \rho_{x}
$$

If we consider that the system is not too far from the steady state, correspondingly the change in time of the gradient of the density is negligible, we get

$$
v=-b \rho_{x} \cdot t+\hat{c},
$$

where $\hat{c}$ can be related to initial velocity and initial gradients at time $t_{0}$ if these terms do not cancel each other. We suppose that (2.15) holds for a longer time scale than the gradient would undergo of considerable changes. We are interested in a density profile in such a quasi-stationary state. The procedure can be also regarded as taking a first initial condition of the velocity field and iterate it successively [19]. Inserting (2.15) into the mass conservation formula we get

$$
\rho_{t}-t b\left(\rho_{x}\right)^{2}+\hat{c} \rho_{x}-t b \rho \rho_{x x}=0 .
$$

If $\hat{c}=0$ and dividing the equation with $t$ we obtain the following equation

$$
\frac{\rho_{t}}{t}-b\left(\rho_{x}\right)^{2}-b \rho \rho_{x x}=0 .
$$

Dividing the last equation by $b$ and multiplying by 2 , we have

$$
\rho_{t_{1}}-2\left(\rho_{x}\right)^{2}-2 \rho\left(\rho_{x x}\right)=0
$$

where $t_{1}=b t^{2} / 4$. This equation in certain aspects may resemble the Kardar-ParisiZhang(KPZ) equation [18] which describes the dynamics of surface growth. The original KPZ equation (where the third term doesn't contain the $\rho$ variable) was investigated with the self-similar Ansatz by the recent authors [8] and found physically reasonable solutions which can be expressed with the error function.

The last equation can be brought to a more compact form

$$
\rho_{t_{1}}=\left(\rho^{2}\right)_{x x},
$$

There is an even more important relationship with the nonlinear heat conduction or porous media equation which has the form

$$
\rho_{t_{1}}=\left(\rho^{m}\right)_{x x}, \quad m>1 .
$$


In [26] Zeldovich and Kompaneets have found the fundamental solution $\rho_{1}$ of this equation which we write in the following form:

$$
\rho_{1}^{m-1}=t_{1}^{-\alpha(m-1)}\left(A^{2}-B^{2} x^{2} t_{1}^{-2 \beta}\right)_{+}
$$

where $A$ is constant and the subscript + at the bracket indicates that only physically relevant - positive - solutions are taken into account. Regarding the other constants we have

$$
\alpha=\beta=\frac{1}{m+1}, \quad B^{2}=\frac{m-1}{2 m(m+1)} .
$$

One can see that this solution has bounded support in $x$ for any $t_{1}>0$ which is a hyperbolic property. Using comparison principle for such equations one can show this finite speed property for any initial condition having compact support. However, the fronts are not straight lines: $x\left(t_{1}\right)= \pm \frac{A}{B} t_{1}^{\beta}, \beta<1$ so the speed of propagation $\dot{x}\left(t_{1}\right)$ goes to zero if $t_{1}$ goes to infinity. One can also see that $\rho_{1}$ is of source-type: $\rho_{1}(x, 0)=K_{1} \delta(x)$.

In our case $m=2$, consequently $\alpha=\beta=1 / 3$, and the solution for the density distribution reads

$$
\rho\left(x, t_{1}\right)=t_{1}^{-\frac{1}{3}}\left(A^{2}-B^{2} x^{2} t_{1}^{-2 / 3}\right)=\left(\frac{b}{4}\right)^{-\frac{1}{3}} t^{-\frac{2}{3}}\left(A^{2}-B^{2} x^{2}\left(\frac{b}{4}\right)^{-2 / 3} t^{-4 / 3}\right) .
$$

This solution gives us a density profile for a bounded spatial region after sufficiently long time. The velocity field is obtained via 2.15

$$
v(x, t)=\frac{2 x}{3 t} .
$$

For the temperature distribution the

$$
T_{t}+\frac{2 x}{3 t} T_{x}=\lambda T_{x x}
$$

PDE have to be solved. Considering the $T(x, t)=t^{-\gamma} f\left(\frac{x}{t^{\omega}}\right)$ the following ODE is derived

$$
\lambda f^{\prime \prime}-\frac{\eta f^{\prime}}{6}+\gamma f=0
$$

with arbitrary $\gamma$ and for $\omega=1 / 2$. The solutions are the Kummer M and Kummer U functions of the form of

$$
f=c_{1} M\left(\frac{1}{2}-3 \gamma, \frac{3}{2}, \frac{1}{12 \lambda} \eta^{2}\right) \eta+c_{2} U\left(\frac{1}{2}-3 \gamma, \frac{3}{2}, \frac{1}{12 \lambda} \eta^{2}\right) \eta .
$$

The mathematical properties of the Kummer function can be found in [1]. For completeness we give the temperature distribution as well,

$$
T(x, t)=t^{-\gamma}\left[c_{1} M\left(\frac{1}{2}-3 \gamma, \frac{3}{2}, \frac{x^{2}}{12 \lambda t}\right) \frac{x}{\sqrt{t}}+c_{2} U\left(\frac{1}{2}-3 \gamma, \frac{3}{2}, \frac{x^{2}}{12 \lambda t}\right) \frac{x}{\sqrt{t}}\right] .
$$


In contrast to the first case, we applied a simplification in the Euler equation (2.14) therefore we can consider solutions which are outside the scope of the selfsimilar class. Therefore, we may try to find other physically important solutions, like traveling-waves with the Ansatz of $\rho=h(\zeta)=h(x-c t)$ where $\mathrm{c}$ is usually the wave propagation velocity. (We still use $h$ as shape function). However, if we consider (2.17), it can be rewritten in the following form

$$
\rho_{t_{2}}-b\left(\rho_{x}\right)^{2}-b \rho \rho_{x x}=0
$$

where $t_{2}=t^{2} / 2$. We take the

$$
\rho=h(\zeta)=h\left(x-c t_{2}\right)=h\left(x-a t^{2} / 2\right)
$$

Ansatz where instead of the constant propagation speed $c$ one may rather consider a kind of constant acceleration, and the constant $c$ is renotated with $a$. We do this for the purpose of finding analytical solutions, and to get results closer to a physical interpretation.

Inserting this formula into (2.29) we obtain the following ODE:

$$
b h h^{\prime \prime}+h^{\prime}\left(b h^{\prime}+a\right)=0 .
$$

The solution is the following

$$
h(\zeta)=\frac{b c_{1}}{a}\left[\text { Lambert } W\left(\frac{e^{\frac{-b^{2} c_{1}+\zeta a^{2}+c_{2} a}{b^{2} c_{1}}}}{b c_{1}}\right)+1\right]
$$

where $c_{1}, c_{2}$ are integration constants and the Lambert-W function, (which also called the omega function), is the inverse function of

$$
f(W(x))=W(x) e^{W(x)}
$$

and can be evaluated with the following series expansion

$$
W(x)=\sum_{n=1}^{\infty} \frac{(-1)^{n-1} n^{n-2}}{(n-1) !} x^{n} .
$$

Additional properties of this function can be found in [22]. Banwell and Jayakumar [3] showed that a W-function describes the relation between voltage, current and resistance in a diode, and Packel and Yuen [21] applied the W-function to a ballistic projectile in the presence of air resistance. Other applications have been discovered in statistical mechanics, quantum chemistry, combinatorics, enzyme kinetics, the physiology of vision, the engineering of thin films, hydrology, and the analysis of algorithms [15]. Note, that in contrast to other special functions (e.g. Whitakker, Bessel, Kummer functions) the LambertW function is not so widely used or applied. 
The final form of the density field is

$$
\rho\left(x-a t^{2} / 2\right)=\frac{b c_{1}}{a}\left[\text { Lambert } W\left(\frac{e^{\frac{-b^{2} c_{1}+\left(x-a t^{2}\right) a^{2}+c_{2} a}{b^{2} c_{1}}}}{b c_{1}}\right)+1\right] .
$$

Figure 1 presents the density field of (2.35) for the $c_{1}=c_{2}=a=b=1$ parameters. Note, that the solution is a steep wave front.

Inserting the space derivative of the density into (2.15) and multiplying with the time the velocity field reads the following

$$
v\left(x-a t^{2} / 2\right)=-\frac{\text { Lambert } W\left(\frac{e^{\frac{-b^{2} c_{1}+\left(x-a t^{2}\right) a^{2}+c_{2} a}{b^{2} c_{1}}}}{b c_{1}}\right) t}{\text { Lambert } W\left(\frac{e^{\frac{-b^{2} c_{1}+\left(x-a t^{2}\right) a^{2}+c_{2} a}{b^{2} c_{1}}}}{b c_{1}}\right)+1} .
$$

Figure 2 presents the velocity field for the $c_{1}=c_{2}=a=b=1$ parameters. Unfortunately, there is no traveling wave solution for the temperature distribution $T(x, t)=$ $f(\zeta)=f\left(x-a t^{2}\right)$ because the velocity has an explicit time dependence which is transfered to the third equation of (2.1) giving the following expression $0=-\lambda f^{\prime \prime}-$ $2 a t f^{\prime}+v(\zeta) t f^{\prime}$ which still depends on time and cannot be simplified to an ODE of $f(\zeta)$

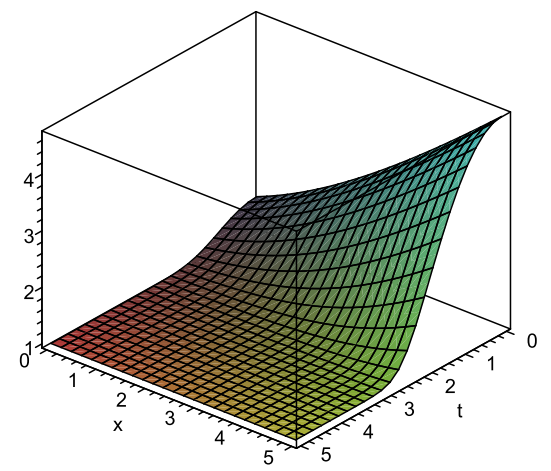

FIGURE 1. The density distribution of Eq. 2.35 for the $a=b=$ $c_{1}=c_{2}=1$ parameters. 


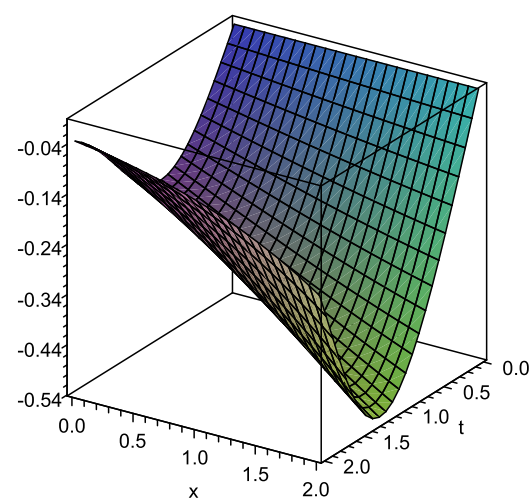

FiguRE 2. The velocity field of Eq. 2.36 for the $a=b=c_{1}=$ $c_{2}=1$ parameters.

\subsection{The linear EOS case}

Let's assume now the linear case of EOS where

$$
p=A \rho
$$

where $A$ is till a constant just to fix the proper physical dimension. From the second case of Eq. (2.1) the velocity field can be obtained for small vs

$$
v=-\frac{A \rho_{x}}{\rho} t \text {. }
$$

From the continuity relation the following PDE is available for the density field

$$
\frac{\rho_{t}}{t}-A \rho_{x x}=0 \text {. }
$$

Note, that the former variable transformation $t_{2}=t^{2} / 2$ is still valid giving us the regular Fourier heat conduction or Fick's diffusion equation of

$$
\rho_{t_{2}}-A \rho_{x x}=0 \text {. }
$$

The solution is the well known Gaussian function of

$$
\rho(x, t)=\frac{1}{\sqrt{A t_{2}}} \exp \left(\frac{-x^{2}}{2 A t_{2}}\right)=\frac{1}{t} \sqrt{\frac{2}{A}} \exp \left(\frac{-x^{2}}{A t^{2}}\right) .
$$

According to (2.38) the velocity field is $v(x, t)=2 x / t$. For the temperature distribution the following PDE have to be solved

$$
T_{t}+2 \frac{x}{t} T_{x}=\lambda T_{x x}
$$


The solution is very similar to the former case of (2.25). Using the same notation the solution reads

$$
f=c_{1} M\left(\frac{1}{2}-\frac{1}{3} \gamma, \frac{3}{2}, \frac{3}{4 \lambda} \eta^{2}\right) \eta+c_{2} U\left(\frac{1}{2}-\frac{1}{3} \gamma, \frac{3}{2}, \frac{3}{4 \lambda} \eta^{2}\right) \eta .
$$

For completeness the temperature field is

$$
T(x, t)=t^{-\gamma}\left[c_{1} M\left(\frac{1}{2}-\frac{1}{3} \gamma, \frac{3}{2}, \frac{3 x^{2}}{4 \lambda t}\right) \frac{x}{\sqrt{t}}+c_{2} U\left(\frac{1}{2}-\frac{1}{3} \gamma, \frac{3}{2}, \frac{3 x^{2}}{4 \lambda t}\right) \frac{x}{\sqrt{t}}\right] .
$$

Figure 3 presents the Kummer M part of the whole solution with $c_{1}=\gamma=\lambda=1$, and $c_{2}=0$ parameters. The Kummer $\mathrm{U}$ part looks very similar. It is straightforward that the PDE of the density field (2.39) can be investigated with the traveling wave Ansatz (2.30), too. The resulting is the next linear ODE

$$
A h^{\prime \prime}-a h^{\prime}=0 \text {. }
$$

The solution is obvious $h=c_{1}+c_{2} e^{\frac{a \zeta}{A}}$ with the density field of $\rho(x, t)=c_{1}+$ $c_{2} e^{\frac{a\left(x-a^{2} t / 2\right)}{A}}$. It turns out from (2.38) that $v=-a t$. After trivial calculations from the heat conduction equation we get $T(x, t)=c_{1}+c_{2}\left(x-a t^{2} / 2\right)$.

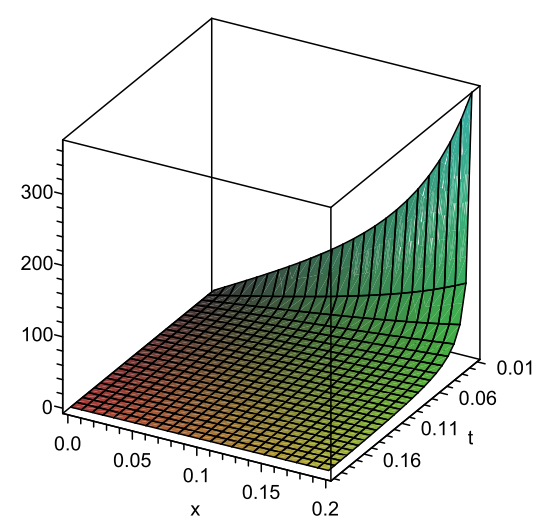

FIGURE 3. The temperature field of Eq. 2.44 only the Kummer M function is presented with the $\gamma=\lambda=c_{1}=1$ parameters.

\subsection{Investigation of the virial EOS}

We still consider the original system of (2.1), and change the EOS in the second equation. Outside the ideal gas and the polytropic equation of state the most important material law is the Van der Waals (VdW) formula for gases where $p(\rho, T)=$ 
$\frac{T \rho}{a-\rho}-b \rho^{2}$ where two of the constants $(a, b)$ can be calculated from the critical point. One of them a is proportional to the universal gas constant times the molar mass of the fluid. Plugging this expression back to the Euler equation and calculating the derivatives we get constrains to the exponents of the Ansatz $\alpha, \beta, \gamma$. For VdW EOS these constrains are contradictory, so no ODEs can be obtained, no self-similar solution can be evaluated.

We may go further and try the virial expansion which can be written in various forms, one possibility is the following

$$
p=A T \rho\left(1+B \rho+C \rho^{2}\right)
$$

where $A, B, C$ are constants and can be fixed from experiments. We try the simplest $B=C=0$ case. Apply the Ansatz of Eq. (2.3). The universality relations are therefore $\alpha=1, \beta=\gamma=\delta=1 / 2$, which dictate the next ODE system

$$
\begin{aligned}
-\frac{h}{2}-\frac{1}{2} \eta h^{\prime}+g h^{\prime}+h g^{\prime} & =0, \\
-\frac{1}{2} g-\frac{1}{2} \eta g^{\prime}+g g^{\prime} & =-A f^{\prime}-\frac{A f h^{\prime}}{h}, \\
-f-\frac{1}{2} \eta f^{\prime}+g f^{\prime} & =\lambda f^{\prime \prime} .
\end{aligned}
$$

Note, that the first equation is a total derivate and can be integrated immediately which fixes $g=\eta / 2$ as for the first investigated system in the Eq. (2.5). So, $v(x, t)=$ $\frac{x}{2 t}$. (We fixed the integration constant to zero.) Inserting this into the second and third equation of (2.47) we get

$$
\begin{aligned}
-\frac{\eta}{4} & =-A f^{\prime}-\frac{A f h^{\prime}}{h}, \\
-f & =\lambda f^{\prime \prime} .
\end{aligned}
$$

The solution for $f$ reads:

$$
f(\eta)=c_{1} \sin \left(\frac{\eta}{\sqrt{\lambda}}\right)+c_{2} \cos \left(\frac{\eta}{\sqrt{\lambda}}\right)
$$

which means

$$
T(x, t)=\frac{1}{t}\left[c_{1} \sin \left(\frac{x}{\sqrt{\lambda t}}\right)+c_{2} \cos \left(\frac{x}{\sqrt{\lambda t}}\right)\right]
$$

Finally, the formal solution for $h(\eta)$ for the density shape function can be derived

$$
h(\eta)=c_{3} \int \cdot \frac{-A c_{1} \cos \left(\frac{z}{\sqrt{\lambda}}\right)+A c_{2} \sin \left(\frac{z}{\sqrt{\lambda}}\right)+\frac{z \sqrt{\lambda}}{4}}{\sqrt{\lambda} A\left[c_{1} \sin \left(\frac{z}{\sqrt{\lambda}}\right)+c_{2} \cos \left(\frac{z}{\sqrt{\lambda}}\right)\right]} d z .
$$


For given parameters like $c_{1}=0, c_{2}=c_{3}=A=\lambda=1$ the integral can be evaluated in a closed form of

$$
h(\eta)=\frac{c_{3}\left(1+i e^{i \eta}\right)^{-\frac{\eta}{4}}\left(1-i e^{i \eta}\right)^{\frac{\eta}{4}} e^{-\frac{i}{4}\left[-4 \eta-d i \log \left(1+i e^{i \eta}\right)+\operatorname{dilog}\left(1-i e^{i \eta}\right)\right]}}{e^{2 i \eta}+1}
$$

where $i$ is the complex unit and $\operatorname{dilog}(x)$ is the dilogarithm (or Spence's) function defined via the following integral

$$
\operatorname{dilog}(x)=-\int_{0}^{x} \frac{\ln |1-s|}{s} d s .
$$

A detailed mathematical description of the properties of the dilogarithm function can be found in [1]. For completeness we give the final formula of the density

$$
\begin{gathered}
\rho(x, t)=t^{\frac{-1}{2} \times} \\
{\left[\frac{\left(1+i e^{i x / \sqrt{t}}\right)^{-\frac{x / \sqrt{t}}{4}}\left(1-i e^{i x / \sqrt{t}}\right)^{\frac{x / \sqrt{t}}{4}} e^{-\frac{i}{4}\left[-4 x / \sqrt{t}-\operatorname{dil} \log \left(1+i e^{i x / \sqrt{t}}\right)+\operatorname{dilog}\left(1-i e^{i x / \sqrt{t}}\right)\right]}}{e^{2 i x / \sqrt{t}}+1}\right]}
\end{gathered}
$$

Note, that the velocity and the temperature distributions in the first and the last model are very similar to each other.

\section{SUMMARY AND OUTLOOK}

We investigated the basic one-dimension coupled PDE equations describing fluid flow with heat conduction and presented self-similar solutions for fluid density, flow velocity and temperature. In certain cases we have tried to find solutions for the system of equation presented in (2.1). In the case when $p \sim \rho^{3}$ the major achievement is that an exact solution is available and the long time decay of density and pressure is a power law of the time variable. The other situation when $p \sim \rho^{2}$ is more complicated from the point of view of exact solutions, consequently we tried to arrive to results iteratively. In this case solutions related to waves has been also obtained with the help of a special function. The following case when $p \sim \rho$ is again a nontrivial situation in the frame of eq. (2.1). At this point one can arrive to solutions iteratively, which in certain aspects may resemble the diffusivity in dilute systems. Moreover the long time behavior a kind of power law decay for density and pressure can be also observed.

Further possible study may be the investigation of the system (2.1), with other possible equations of state, which may be different than the ones considered here. The extension of Eq. (2.1) for real fluids (e.g. including a viscous term, temperature or density dependent viscosity etc.) and searching for possible analytic or eventually exact solutions is also a possible future problem to be investigated. Regarding computational methods we hope that our study can help to develop benchmark tests for various numerical codes or models. 


\section{ACKNOWLEDGEMENT}

We thank for Prof. Robert Kersner for useful discussions.

\section{REFERENCES}

[1] M. Abramowitz and I. Stegun, Handbook of Mathematical Functions, 2nd ed. Dover, New York,: John Wiley \& Sons, 1968, vol. III.

[2] S. N. Aristov and A. D. Polyanin, "New classes of exact solutions and some transformations of the Navier-Stokes equations," Russ. J. Math. Phys., vol. 17, no. 1, pp. 1-18, 2010.

[3] T. C. Banwell and A. Jayakumar, "Exact analytical solution for current flow through diode with series resistance," Elect. Lett., vol. 36, no. 1, pp. 29-33, 2000.

[4] G. I. Barenblatt, Similarity, self-similarity, and intermediate asymptotics. Transl. from Russian by Norman Stein, M. Van Dyke, Ed. New York, London: Consultants Bureau, 1979.

[5] I. F. Barna, "Self-similar solutions of three-dimensional Navier-Stokes equation," Commun. Theor. Phys., vol. 56, no. 4, pp. 745-750, 2011.

[6] I. F. Barna and R. Kersner, "Heat conduction: a telegraph-type model with self-similar behavior of solutions," J. Phys. A, Math. Theor., vol. 43, no. 37, p. 6, 2010.

[7] I. F. Barna and R. Kersner, "Heat conduction: hyperbolic self-similar shock-waves in solids," accepted in Shock Waves, Preprint Server http://arxiv.org/abs/1204.4386.

[8] I. F. Barna and L. Mátyás, "Self-similar solution of the kardar-parisi-zhang interface dynamic equation," Preprint Server http://arxiv.org/abs/1112.2870?context=nlin.AO.

[9] K. Fakhar, T. Hayat, Y. Cheng, and N. Amin, "A note on similarity solutions of Navier-Stokes equations," Commun. Theor. Phys., vol. 53, no. 3, pp. 575-578, 2010.

[10] V. I. Fushchich, V. M. Shtelen, and S. L. Slavutskij, "Reduction and exact solutions of the NavierStokes equations," J. Phys. A, Math. Gen., vol. 24, no. 5, pp. 971-984, 1991.

[11] B. H. Gilding and R. Kersner, Travelling waves in nonlinear diffusion-convection reaction, ser. Progress in Nonlinear Differential Equations and their Applications. Basel: Birkhäuser, 2004, vol. 60.

[12] G. Gradenigo, A. Sarraceno, D. Villamaina, and A. Puglisi, "Fluctuating hydrodynamics and correlation lengths in a driven granular fluid," J. Stat. Mech., vol. P08017, 2011.

[13] V. Grassi, R. A. Leo, G. Soliani, and P. Tempesta, "Temperature behavior of vortices of a 3D thermoconducting viscous fluid," Physica, vol. 286, pp. 79-84, 2000.

[14] V. N. Grebenev, M. Oberlack, and A. N. Grishkov, "Lie algebra methods for the applications to the statistical theory of turbulence," J. Nonlinear Math. Phys., vol. 15, no. 2, pp. 227-251, 2008.

[15] B. Hayes, "Why W?" Amer. Sci., vol. 93, pp. 104-109, 2005.

[16] X. R. Hu, Z. Z. Dong, and F. Huang, "Reductions and exact solutions of the (2+1)-dimensional Navier-Stokes equations," Z. Naturforschung A., vol. 65, pp. 504-509, 2010.

[17] X. Jiao, "Some similarity reduction solutions to two-dimensional incompressible Navier-Stokes equation," Commun. Theor. Phys., vol. 52, no. 3, pp. 389-394, 2009.

[18] M. Kardar, G. Parisi, and Y. Zhang, "Dynamical scaling of growing interfaces," Phys. Rev. Lett., vol. 56, pp. 889-892, 1986.

[19] G. Micula and P. Pavel, Differential and integral equations through practical problems and exercises, ser. Kluwer Texts in the Mathematical Sciences. Dordrecht: Kluwer Academic Publishers, 1992, vol. 7.

[20] M. Nadjafikhah, "Group analysis of three dimensional Euler equations of gas dynamics," Preprint Server http://arxiv.org/abs/0908.3598.

[21] E. Packel and D. Yuen, "Projectile motion with resistance and the Lambert W function," College Math. J., vol. 35, pp. 337-341, 2004. 
[22] G. Pólya and G. Szegö, Aufgaben und Lehrsätze aus der Analysis. Bd. I: Reihen, Integralrechnung, Funktionentheorie. Bd. II: Funktionentheorie, Nullstellen, Polynome, Determinanten, Zahlentheorie. S. Berlin: Julius Springer. (Die Grundlehren der mathematischen Wissenschaften in Einzeldarstellungen. Bd. XIX, XX., 1925.

[23] L. Sedov, Similarity and Dimensional Methods in Mechanics. CRC Press, 1993.

[24] J. C. Tannehill, D. A. Anderson, and R. H. Pletcher, Computational Fluid Mechanics and Heat Transfer. Washington: Taylor and Francis, 1987.

[25] M. Yuen, "Analytical solutions to the Navier-Stokes equations," J. Math. Phys., vol. 49, no. 11, pp. $113102,10,2008$.

[26] Y. B. Zel'dovich and A. S. Kompaneets, "Collection dedicated to the 70th birthday of A. F. Joffe," Izdat. Akad. Nauk SSSR, p. 61, 1950.

[27] Y. B. Zel'dovich and Y. P. Raizer, Physics of Shock Waves and High Temperature Hydrodynamic Phenomena. New York: Academic Press, 1966.

[28] O. C. Zienkiewicz, R. L. Taylor, and P. Nithiarasu, The finite element method for fluid dynamics, 6th ed. Amsterdam: Elsevier Butterworth-Heinemann, 2005.

Authors' addresses

Imre Ferenc Barna

Wigner Research Center of the Hungarian Academy of Sciences, H-1525 Budapest, P.P. Box 49, Hungary

Current address: Wigner Research Center of the Hungarian Academy of Sciences, H-1121 Budapest, Konkoly-Thege út 29-33, Hungary

E-mail address: barna.imre@wigner.mta.hu

László Mátyás

Sapientia University, Department Bioengineering, Libertătii sq. 1, 530104 Miercurea Ciuc, Romania Current address: Sapientia University, Department Bioengineering, Libertătii sq. 1, 530104 Miercurea Ciuc, Romania

E-mail address: matyaslaszlo@sapientia.siculorum.ro 